\title{
Measuring entropy in functional neuroscience: pathophysiological and clinical applications
}

This article was published in the following Dove Press journal:

Neuroscience and Neuroeconomics

26 July 2016

Number of times this article has been viewed

\section{Chen-Chih Chung' \\ Jiunn-Horng Kang ${ }^{2,3}$ \\ Chaur-Jong $\mathrm{Hu}^{\prime}$}

'Department of Neurology, Shuang Ho Hospital, College of Medicine,

Taipei Medical University, New Taipei, ${ }^{2}$ Department of Physical Medicine and Rehabilitation, Taipei Medical University Hospital, ${ }^{3}$ Department of Physical Medicine and Rehabilitation, School of Medicine, College of Medicine, Taipei Medical University,

Taipei, Taiwan, Republic of China
Correspondence: Chaur-Jong $\mathrm{Hu}$ Department of Neurology, Shuang Ho Hospital, College of Medicine, Taipei Medical University, 29I, Jhongjheng Road, Jhonghe District, New Taipei 235, Taiwan, Republic of China Email chaurjongh@s.tmu.edu.tw
Abstract: A biological system obtains information, reacts to stimuli, and modifies its behavior to adapt to the environment via complex control systems. A healthy system is expected to adequately adapt to a variety of changes. Physiological signals obtained from a healthy individual should contain rich information and complex behaviors. Entropy-derived measures have been used to access the complexity of the physiological signals. Aging or diseased status usually shows reduced entropy values and loss of complexity within the dynamics of physiological output. In this article, we aim to review the available evidence related to the pathophysiological nature of complexity and the clinical applications of entropy-derived measures in varied neurological disorders.

Keywords: complexity, entropy, nerve system, electroencephalography, pathophysiology

\section{Introduction}

"Survival of the fittest". Evolution is characterized as the change in traits of biological populations over generations. In order to survive, an organism or a biological system needs to adapt to its environment by using the information of itself and the environment to modify behaviors and to solve the life history problems. ${ }^{1}$ The capacity of adaptation involves detecting, responding, and reacting to the innumerable perturbations of daily life. ${ }^{1}$ This capacity is achieved via complex integration of internal and external stimuli and interactions between multiple control systems and is regulated at scales of different orders of magnitude in space and time. In general, biological system exhibits a hierarchical structure: genes encode proteins, proteins constitute organelles and cells, and cells form tissues and organs. ${ }^{2,3}$ The space ranges from molecular to the living organism scale. At the molecular scale, the dynamics is dominated by random and short-timescale fluctuations, while at organ or organism scale, the dynamics is more deterministic with longer timescale. ${ }^{4}$ Thus, the timescale could range from nanoseconds to years in a biological system. Regulation of this complex system over different temporospatial scales via both bottom-up and top-down directions forms a feedback loop and contains rich information of the control system. The fitness of an organism depends on the richness of the information that is available and the complexity of the structural and regulatory systems, and an organism has to react with its internal or external needs and to interact with one another. ${ }^{2}$ How to measure and describe the complexity of a biological system has been drawing attention in recent years. Many efforts have been made to quantify and distinguish the health and different diseases via the measure of complexity. ${ }^{3-6}$ Among the 
methods of quantifying the complexity of a system, entropy analysis was introduced.

\section{Pathophysiological aspects of entropy}

The concept of entropy was introduced and developed in thermodynamics in the early 1850 s by Rudolf Clausius, in response to the observation that a certain amount of functional energy released from combustion reactions is always lost to dissipation or friction. In 1877, Ludwig Boltzmann developed a statistical mechanical evaluation of entropy and considered entropy as a measure of statistical disorder. Entropy increases with the degree of disorder and is maximal for completely random systems. ${ }^{5}$ In the $1940 \mathrm{~s}$, Shannon ${ }^{7}$ applied the concept of entropy in communication theory to evaluate the statistical nature of "lost information" in phone-line signals. In the communication theory, the amount of information transmitted is the amount of information that is missing in the received signal or, alternatively, the uncertainty of a signal of what was actually sent when it is received. ${ }^{7}$ Entropy-based algorithms are then developed to be a quantitative measurement of the uncertainty. Complexity measured by entropy evaluates the amount of information needed to predict the future state of the system, and more complex dynamics are represented as larger entropy. ${ }^{8}$

In recent years, the concept of entropy-derived measures has been used in analyzing physiological signals in the studies evaluating the complexity of biological systems.

A healthy system is expected to adequately adapt to a variety of changes. Therefore, physiological signals obtained from a healthy individual should contain rich information and complex behaviors. On the contrary, aging and diseased status, usually implying decrease of the richness of physiological information and less fit to the environment, show reduced entropy values and loss of complexity within the dynamics of physiological output. ${ }^{3,6}$ The loss of physiological complexity is believed to result from deterioration of underlying structural components of physiological systems, as well as impairments within the coupling between these systems. ${ }^{1,3}$ For now, entropy-derived analyses have been widely applied in numerous studies and clinical usages, demonstrating the disease-related loss of physiological complexity in the dynamics of varied physiological systems, including cardiovascular, ${ }^{8-10}$ respiratory, ${ }^{11-13}$ and neurological systems. ${ }^{14-16}$

\section{Different entropy-based metrics}

Numerous entropy metrics are available to quantify different aspects of the complexity of physiological signals (Figure 1).
Approximate entropy (ApEn) and its modified methods are sets of measures of system complexity closely related to entropy, were introduced to apply in electrocardiogram, and are widely used to analyze heart rate-related data in clinical cardiovascular studies, as well as endocrine hormone release pulsatility. ${ }^{17,18}$

ApEn is applicable to noisy, typically short, real-world time series and can distinguish between correlated stochastic processes. ${ }^{19}$

Sample entropy (SampEn) is a modified algorithm of ApEn, when the bias of self-matches is avoided. As a result, it represents an estimator that is largely independent of the length of the time series. ${ }^{19}$ SampEn has been applied in electroencephalogram (EEG) and brain magnetic resonance imaging (MRI) data to evaluate the complexity of nonlinear structures in brain dynamics. ${ }^{16,19}$ These entropy-based metrics are based on a "one-step difference" and estimate the degree of irregularity of a time series over a single scale of time. Hence, they do not account for features related to structure and organization over a range of timescales. ${ }^{19}$ However, as the hierarchical structure of a real biological system exhibits different space and timescales, and the interactions can occur at the same scale as well as between scales, the traditional entropy that measures one single timescale may not be able to characterize and capture the whole structure of the complexity of a real biological system.

In order to overcome this limitation and to investigate the variability in physiological signals across multiple temporal scales, numerous metrics were developed, including multiscale entropy (MSE) analyses ${ }^{5}$ and multivariate MSE. ${ }^{20,21}$ These methods allow the detection of multiple, hierarchic levels, and complex fluctuation of neurophysiological output signal of a biological system. Furthermore, the goal of multiscale modeling is not only to model a system at multiple scales but also to conserve the information from a lower scale to a higher scale, so that the information from the very bottom scale can be carried to the top scale correctly. ${ }^{4}$

MSE method, as described by Costa et al, ${ }^{5}$ aims at quantifying the interdependence between entropy and timescale, enabled by evaluating entropy of univariate time series, a technique called "coarse-graining" at multiple temporal scales. This is associated with the ability of living systems to adjust to a changing environment. The underlying integrative multiscale functionality is interpreted by nondiminishing entropy values across increasing timescales. ${ }^{19} \mathrm{MSE}$ has the advantage of facilitating the assessment of the dynamical complexity of a system and being applicable to both physiological and physical signals of finite length. ${ }^{22}$ 


\begin{tabular}{|c|c|}
\hline $\begin{array}{l}\text { Recording } \\
\text { conditions }\end{array}$ & $\begin{array}{ll} & \text { Organization of } \\
\text { Noise } & \text { neuronal system }\end{array}$ \\
\hline \multicolumn{2}{|c|}{ Signal processing and mathematic characteristic } \\
\hline $\begin{array}{l}\text { Frequency domain approaches (eg, FFT, HHT) } \\
\text { decomposition of signals } \\
\text { into different frequency bands }\end{array}$ & $\begin{array}{l}\text { Entropy approaches (eg, ApEn, SampEn, MSE) } \\
\text { usually taking negative logarithm of probabilities of } \\
\text { specific conditions based on self-similarity or } \\
\text { randomness of signals }\end{array}$ \\
\hline $\begin{array}{l}\text { Physiological } \\
\text { basis }\end{array}$ & $\sqrt{t}$ \\
\hline $\begin{array}{l}\text { Neuronal activity is mainly frequency } \\
\text { coded and modulated }\end{array}$ & $\begin{array}{l}\text { The complexity of specific biological system involved } \\
\text { and encoded in the signals }\end{array}$ \\
\hline \multicolumn{2}{|l|}{ Advantages } \\
\hline 1. Easy visualization of relevant concepts & $\begin{array}{l}\text { 1. Can describe the nonlinear behaviors of the } \\
\text { system which may be difficult to be studied with } \\
\text { traditional approaches }\end{array}$ \\
\hline $\begin{array}{l}\text { 2. Frequency (eg, neuronal firing rate) } \\
\text { can be easily observed in basic research }\end{array}$ & $\begin{array}{l}\text { 2. Seem to be more sensitive in specific } \\
\text { pathological conditions than traditional } \\
\text { approaches }\end{array}$ \\
\hline \multicolumn{2}{|l|}{ Disadvantages } \\
\hline $\begin{array}{l}\text { 1. Most of biological signals are nonlinear and } \\
\text { nonstationary which may hamper the results }\end{array}$ & $\begin{array}{l}\text { 1. No standardized procedure regarding the } \\
\text { computation of complexity }\end{array}$ \\
\hline & $\begin{array}{l}\text { 2. The physical explanation and correlation with } \\
\text { basic biological system is still unknown }\end{array}$ \\
\hline
\end{tabular}

Figure I The characteristics of the methods in biosignal processing.

Abbreviations: ECG, electrocardiography; EEG, electroencephalogram; EMG, electromyography; FFT, fast Fourier transform; HHT, Hilbert-Huang transform; ApEn, approximate entropy; SampEn, sample entropy; MSE, multiscale entropy.

Multivariate MSE evaluates SampEn over different timescales and deals with the different embedding dimensions, time lags, and amplitude ranges of data channels in a rigorous and unified way. The method is shown to cater for linear and/or nonlinear within- and cross-channel correlations as well as for complex dynamical couplings and various degrees of synchronization over multiple scales, thus allowing for direct analysis of multichannel data.

\section{Clinical applications of entropy in neuroscience}

The human neurological systems comprise complex structural architecture and functional communication networks. ${ }^{1,22}$ In the past, the function of neuronal circuit has been mainly conceptualized by linear modelization and failed to fully describe the dynamics of the neurophysiological signals. A large and growing body of studies has applied the nonlinear analyses, such as entropy-derived measures for evaluating the physiological complexity in neuroscience.

\section{Autonomic Nervous system}

The applications of entropy on human physiological signals were developed earlier for analyzing the heart rate and beatto-beat blood pressure. ${ }^{8}$ Heart rate is influenced by numerous factors including the liquid metabolism, hormonal and temperature variations, physical activity, circadian rhythms, and autonomic nervous system. As a result, heart rate variations are extremely complex in healthy individuals. ${ }^{8}$ Aging and diseases, such as atrial fibrillation (AF) and heart failure, have been associated with reduced complexity in heart rate variability (HRV) and greater mortality. ${ }^{5}$ The entropy of heart rate was linked to neurological system since the modulation of heart beat was associated with the two components of autonomic nervous system: sympathetic and parasympathetic 
nerves. ${ }^{23}$ Rhythmical oscillations of both heart rate and blood pressure have been indicated to reflect the sympathetic and parasympathetic modulation. ${ }^{24,25} \mathrm{HRV}$ was proposed to be a reliable tool to evaluate the function of the autonomic nervous system. ${ }^{26}$

Using ApEn, Li et al analyzed the 24-hour HRV signals from a total of 29 healthy controls and 63 type 2 diabetes patients. ApEn values extracted from low- and high-frequency components of HRV of healthy controls were higher than those of diabetes patients, suggesting lower complexity of the cardiovascular system and worse regulation function in response to changes of environment in individuals with diabetes when compared with healthy controls. ${ }^{27}$ Sungnoon et $\mathrm{al}^{28}$ used SampEn to evaluate the signal complexity of HRV extracted from surface electrocardiography (ECG) in control subjects and individuals with AF. Their results showed that SampEn values from lead V1 in patients with persistent AF were significantly higher than those in healthy controls. The low-frequency to high-frequency power ratio of HRV analysis in both paroxysmal AF and persistent AF patients was also significantly decreased when compared to healthy controls. The data reflected the impairment of cardiac autonomic function in both paroxysmal AF and persistent AF patients consistent with an increased atrial signal irregularity.

Using the MSE method, Turianikova et $\mathrm{al}^{29}$ investigated the effect of orthostatic challenge on the complexity of HRV and blood pressure variability and the correlation between MSE and traditional linear (time and frequency domain) measures in 28 healthy young individuals. They found that MSE of HRV and blood pressure variability signals is sensitive to changes in autonomic balance caused by postural change from the supine to the standing position, and the effect of orthostatic challenge on complexity depended on the timescale of MSE. ${ }^{29}$

Alteration of autonomic nervous system in acute hypoxia was noted when evaluating the cardiovascular dysfunction with SampEn by Zhang et al, ${ }^{30}$ when they investigated eight healthy male subjects exposed to simulated altitude from sea level to $3,600 \mathrm{~m}$ in 10 minutes. The results showed that hypoxia attenuated both SampEn and spectral HRV parameters, with the shift of balance of sympathovagal toward sympathetic dominance.

Renyi entropy and ApEn have also been proposed to be used to detect early cardiac autonomic dysfunctions. ${ }^{31,32}$ Significant difference of Renyi entropy derived from a 20-minute, lead-II ECG recording between controls and early or definite cardiac autonomic neuropathy in diabetes patients forms a useful tool to detect the autonomic impairment in the early stages of the disease and has the potential to improve treatment and outcomes. ${ }^{31}$

Early assessment of the complexity of HRV by MSE has shown its value of predicting stroke-in-evolution and outcomes in acute ischemic stroke patients. ${ }^{33,34}$

Entropy values over single or different timescales may reflect certain behavior of heart rate dynamics and have the implications of the complexity of autonomic nervous system. Abnormality of complexity revealed by HRV analyzed by entropy method could be a sensitive indicator of autonomic dysfunction and suggests underlying impairment of interaction in the brain-heart axis. ${ }^{34}$

\section{Sleep regulation}

The sleep process is also involved in the regulation of autonomic nervous system. The transition from wake to sleep and between different sleep stages has been shown to be characterized by the activation of several cortical, subcortical, and brainstem neural circuits. The processes also involve in changes in many hormones, neurotransmitters, internal circadian rhythms, and environmental variations. ${ }^{35}$ Observation of the fluctuations between sympathetic and parasympathetic predominance upon sleep onset and during the transitions between different sleep stages reveals that the cardiovascular system is strongly affected by different sleep stages. From sleep stages N1 to N3, a gradual decrease in heart rate and blood pressure could be observed, and during rapid eye movement (REM) sleep, activation of cardiovascular system is dominant, and sometimes to the level similar or higher than wakefulness. ${ }^{36}$

Measuring complexity of HRV during sleep has been used to evaluate the regulation of autonomic nervous system in physiological and pathological sleep. An earlier study has shown that in healthy adults, non-REM (NREM) sleep is characterized by an increased SampEn of HRV, and the SampEn of HRV during REM sleep was similar to wake status (Wake: 1.53 \pm 0.28 , NREM: 1.76 \pm 0.32 , REM: 1.45 \pm 0.19 , $P=0.005) .{ }^{37}$ Differences between the HRV analyzed by using the ApEn in postmenopausal women have shown the specific features in heart rate dynamics during various sleep stages. ${ }^{38}$

Decreased physiological complexity of HRV during sleep by using entropy method has been associated with aging-related process. In the study presented by Viola et al, who evaluated the HRV in polysomnography of 12 healthy young $(21.1 \pm 0.8$ years $)$ and 12 healthy older subjects (64.9 \pm 1.9 years), the older adults showed a significant reduction of Shannon entropy and corrected conditional entropy. And the decrease of entropy measures was more evident during REM sleep. ${ }^{39}$ 
With using the MSE method to measure the physiological complexity of sleep under different time scale factors, Yang et al reported the altered autonomic function in patients with depression and insomnia. ${ }^{40}$ Compared with the controls, the patients with major depression and primary insomnia exhibited significant reductions in parasympathetic-related HRV indices and also exhibited significant reductions in physiological complexity during the sleep period. An earlier study focused on the complexity metrics of the HRV during sleep in patients with obesity showed that the obesity group presented significantly reduced complexity during light and deep sleep (deep sleep stage III, obese group: SampEn $=1.34 \pm 0.56$; controls group: SampEn $=1.68 \pm 0.4$; sleep stage II, obese group: $S a m p E n=1.27 \pm 0.41$; controls group: SampEn $=1.54 \pm 0.38, P \leq 0.05) .{ }^{41}$ Entropy-based HRV parameters have been suggested to improve the classification of different sleep stages. ${ }^{42}$

Entropy-based analysis of EEG during sleep has also been applied in various physiological status and disorders. The work by Burioka et $\mathrm{al}^{43}$ has shown that the ApEn values for EEG signals were lower during stage IV sleep and higher during wake and REM sleep $(0.896 \pm 0.264$ during eyes-closed waking state, $0.738 \pm 0.089$ during Stage I, $0.615 \pm 0.107$ during Stage II, $0.487 \pm 0.101$ during Stage III, $0.397 \pm 0.078$ during Stage IV, and $0.789 \pm 0.182$ during REM sleep) in eight healthy young adults. As in entropybased HRV analysis, entropy for EEG has been suggested to be able to be used as a criterion for sleep staging ${ }^{44}$ and in evaluation of consciousness status. ${ }^{45,46}$ Parkinson's disease (PD), which is known to be related to various sleep disorders during the progressive course of the disease and commonly have negative effects on the patients' quality of life, has been linked to altered MSE of EEG during NREM sleep. ${ }^{47}$ These results suggested that entropy-derived measures are useful tools to detect and provide important information of the complexity of autonomic cardiovascular and neurophysiological controls during sleep and also indicated that the reduction of entropy in diseased status and aging is characterized by impairment of cardiac control mechanisms. Sleep is a highly regulated behavior involving complex neural networks and neurotransmitters. The mechanism of neuronal circuits regulating sleep process and its correlation to different measures of entropy value are not fully understood; altered complexity of physiological signals during sleep may reflect the nature of impaired neuronal connectivity. Entropy values of different temporospatial scales possibly represent the hierarchical composition of the nervous systems. Further research to clarify the pathophysiological mechanisms underlying the altered complexity during sleep are warranted to improve the understanding of the associations between different diseases and sleep disorders.

\section{Alzheimer's disease and cognitive dysfunction}

Alzheimer's disease (AD) is characterized by progressive deterioration of cognitive function and behavioral disturbance. The neuropathological features of AD include generalized neuronal cell loss, neurofibrillary tangles, and senile plaques in brain regions. EEG has been widely employed as a noninvasive clinical tool for examining normal and pathologic, neurophysiological temporal dynamics. Nonlinear EEG complexity analyses have been applied to understand the physiological processes in patients with dementia. Several studies demonstrated the utility of MSE in evaluating EEG changes in AD. Using ApEn ${ }^{48,49}$ and SampEn, ${ }^{50}$ reduced complexities in EEG signals in AD have been reported. ${ }^{51,52}$ With regard to MSE measures, studies have found lower EEG complexity in $\mathrm{AD}$ patients compared to that of age-matched controls, ${ }^{53,54}$ and increased MSE value at higher scale factors has been associated with the decline in cognitive function in $\mathrm{AD}$, as determined by mini-mental state examination scores. MSE complexity of EEGs in various brain areas has also been reported to correlate to subdomains of neuropsychiatric functions. ${ }^{55}$ The pathological presentations of $\mathrm{AD}$, including neuronal loss and deficiency of neurotransmitters, result in altered corticocortical association fibers and cause disconnection syndrome. ${ }^{56}$ The complexity of EEGs at different time scales might represent neuronal network connectivity of the brain of AD patients. The neurophysiological mechanism underlying the contradiction between decreased EEG complexity across smaller scale factors (shorter time scales) and increased complexity at larger scale factors (longer time scales) for severe $\mathrm{AD}$ patients globally across brain regions remains not clearly understood. It may reflect the changes of brain dynamics under the process of $\mathrm{AD}$. A recent study using functional magnetic resonance imaging (fMRI) to evaluate the integrity of brain systems sustaining cognition has observed a decrease in anteroposterior interactions and, in contrast, increased interactions within the frontal and parietal subsystems in the elderly compared to young controls. ${ }^{57}$ This decreased anteroposterior integration was more pronounced in AD patients compared to elderly controls, particularly in the precuneus-posterior cingulate region. Whether the diversity of entropy measures at different timescales could be 
explained by the changes in the interactions between different brain regions, or it reflects the hierarchical composition of the central nervous system, still warrants elucidation.

Entropy measures of EEG complexity have also been applied in many other pathological states, including toxic states,${ }^{58}$ seizure, ${ }^{59}$ schizophrenia, ${ }^{60,61}$ and anesthesia. ${ }^{62,63}$

\section{Parkinson's disease}

PD is a degenerative disorder of the central nervous system affecting mainly the motor and various nonmotor systems. Pathological features include degeneration of dopaminesecreting cells in the substantia nigra pars compacta of basal ganglia coupled with intracytoplasmic inclusions known as Lewy bodies. ${ }^{64}$ Reduced dopaminergic neuronal activity caused by cell death results in disruption of the pathways connecting basal ganglia with different brain regions, such as motor, oculomotor, associative, limbic, and orbitofrontal areas. Disconnection of these circuits involved in a wide variety of functions explains the varied symptoms of the disease. Previous study has shown that the irregularity in the neuronal firing activity of EEG, muscular activity of electromyography (EMG), and kinetics of movement exhibit a complex temporal organization in PD. ${ }^{65}$ Using the entropy methods, many studies have reported the changes in the complexity of physiological signals in PD, including lower ApEn of Parkinsonian tremor on EMG activities, altered entropy of gait rhythms, and lower SampEn of postural stability in PD patients with freezing symptoms. ${ }^{66}$

In PD, changes in EEGs' complexity at awake and during sleep have been reported. With wavelet packet entropy method, EEG signals from patients with PD showed higher entropy over the global frequency domain at resting state. ${ }^{67}$ Sleep stage-specific increases of MSE were observed in PD during NREM sleep. ${ }^{47}$ The changes in the complexity of EEG signals in PD patients may also show the difference of interconnection of the cortical functional island and have been proposed to be used as early diagnosis and early intervention of PD.

From the data stream recorded from globus pallidus internal (GPi) neurons in patients who underwent deep brain stimulation, higher neuronal entropy (as estimated by the ApEn) in the GPi has been reported in the patients with PD than in the patients with dystonia, ${ }^{68}$ indicating a greater irregularity in the neuronal discharge pattern from the output nuclei of the basal ganglia with hypokinetic state. Apomorphine, a nonselective dopamine agonist, when being administered during surgery for deep brain stimulation, has been reported to induce a decrease in entropy measured in the interspike intervals of subthalamic nucleus. In PD, dysfunction in subthalamic nucleus and GPi neuronal data stream generates a large number of different pattern possibilities leading to a signal with limited order and reduced information and enhances the inhibition of motor program by decreasing its informative nature. ${ }^{65}$ Depletion of neuronal activities, reduced richness of information, disorganization of interconnections between neuronal circuitry, and altered global cortical functions may contribute to the changes in the complexity of physiological signals in PD.

\section{Motor control system}

Entropy-derived measures have been applied in evaluating balance and locomotor functions, which are commonly qualified by recording the temporal and spatial fluctuations of physiological signals, such as center of pressure ${ }^{69,70}$ and gait. $^{71,72}$ The normal function of controlling postural sway dynamics and gait is complex, depending on intact integration of musculoskeletal, somatosensory, visual, vestibular, and central nervous systems. It has become evident that aging is associated with diminished postural sway complexity (as quantified by MSE) and independently associated with frailty, low daily activity, and falls in older adults as compared to healthy younger adults. ${ }^{73,74}$ Perturbations, such as visual, somatosensory impairments, ${ }^{75}$ and stressors, such as concurrent cognitive interference, ${ }^{76}$ have been demonstrated to result in decreased complexity of postural control. The center of pressure data and gait measures are often dynamic, nonlinear, and nonstationary in nature. When compared with traditional methods, entropy-derived analyses are shown to be promising and advantageous in analyzing these data. ${ }^{70}$

\section{Other clinical applications}

Several studies have applied entropy-based measures for different physiological signals. Using MSE method for analyzing magnetoencephalography records in patients with traumatic brain injury, lower complexity within multiple brain regions has been demonstrated and correlated with greater variability in cognitive task performance as compared to controls. ${ }^{77}$ In fMRI studies, SampEn of the patients with schizophrenia has more complex fMRI signals than healthy controls. ${ }^{78}$ In the study using blood oxygen level-dependent MRI, an age-related loss of complexity was present in the temporal fluctuations of blood oxygen level-dependent signals from multiple brain regions. ${ }^{79}$ Within older adults, the degree of complexity estimated from numerous brain regions was positively correlated with cognitive functions, including attention, orientation, memory, and verbal fluency. Entropy 
analysis is also feasible to evaluate the associations of the signal complexity between different systems, for instance, the brain and heart connection. Using EEG and 24-hour ECG, MSE analysis showed inverse correlations between the signal complexity of cardiac and cerebral activities. ${ }^{80}$ The resting-awake and fast-photic stimulation EEGs were associated with the R-to-R peak interval of ECG, implying a strong correlation between the dynamics of heartbeat and brainwaves and the link between autonomic network of central nervous system and the modification and control of heart rate.

\section{Rehabilitation and treatment programs}

Entropy-derived methods have also been successfully used and proposed to be sensitive indicators for monitoring and evaluating the effects of different therapies, including rehabilitation, ${ }^{1}$ exercise training ${ }^{81}$ Tai Chi training, ${ }^{82}$ and transcranial direct current stimulation ${ }^{83}$ in the individual with movement disorders or impaired postural control. These treatment-related increases and regains in physiological complexity also correlated closely with functional improvements.

\section{Conclusion}

A growing body of studies has proposed entropy-derived analyses as useful and robust tool to assess the nonlinear physiological signals and evaluate the complexity of a biological system. The use of entropy-derived analyses to describe the physiological signals and neuronal network activities may provide new qualitative and quantitative information relative to the nature of complexity of nervous systems, as well as its distortion in pathological conditions.

It is reasonable to expect that the inclusion of nonlinear features of entropy methods could help with better understanding the complexity and nonstationarity of signals recorded in normal and pathological conditions. Further studies are needed to correlate the results of entropy measures over different temporospatial scales to the functional status and to the assessment of therapeutic effects. The application of entropy-based methods in functional neuroscience provide us new and different perspectives in viewing a biological system and diseases. With these novel tools, new treatments and therapeutic strategies of diseases may be developed by evaluating and optimizing the internal complexity of an organism and also its dynamics to the external environment. The potentials of entropy-based methods have implications for future research and clinical practice.

\section{Author contributions}

All authors contributed toward data analysis, drafting and critically revising the paper and agree to be accountable for all aspects of the work.

\section{Disclosure}

The authors report no conflicts of interest in this work.

\section{References}

1. Manor B, Lipsitz LA. Physiologic complexity and aging: implications for physical function and rehabilitation. Prog Neuropsychopharmacol Biol Psychiatry. 2013;45:287-293.

2. van Baalen M. Biological information: why we need a good measure and the challenges ahead. Interface Focus. 2013;3(6):20130030.

3. Lipsitz LA, Goldberger AL. Loss of "complexity" and aging. Potential applications of fractals and chaos theory to senescence. JAMA. 1992;267(13):1806-1809.

4. Qu Z, Garfinkel A, Weiss JN, Nivala M. Multi-scale modeling in biology: how to bridge the gaps between scales? Prog Biophys Mol Biol. 2011;107(1):21-31.

5. Costa M, Goldberger AL, Peng C-K. Multiscale entropy analysis of complex physiologic time series. Phys Rev Lett. 2002;89(6):068102.

6. Goldberger AL, Peng C-K, Lipsitz LA. What is physiologic complexity and how does it change with aging and disease? Neurobiol Aging. 2002;23(1):23-26.

7. Shannon CE. A mathematical theory of communication. ACM SIGMOBILE Mob Comput Commun Rev. 2001;5:3-55.

8. Kaplan DT, Furman MI, Pincus SM, Ryan SM, Lipsitz LA, Goldberger AL. Aging and the complexity of cardiovascular dynamics. Biophys $J$. 1991;59(4):945-949.

9. Heffernan KS, Fahs CA, Shinsako KK, Jae SY, Fernhall B. Heart rate recovery and heart rate complexity following resistance exercise training and detraining in young men. Am J Physiol Heart Circ Physiol. 2007; 293(5):H3180-H3186.

10. Ji L, Li P, Li K, Wang X, Liu C. Analysis of short-term heart rate and diastolic period variability using a refined fuzzy entropy method. Biomed Eng Online. 2015;14:64.

11. Peng CK, Mietus JE, Liu Y, et al. Quantifying fractal dynamics of human respiration: age and gender effects. Ann Biomed Eng. 2002;30(5): 683-692.

12. Faes L, Widjaja D, Van Huffel S, Nollo G. Investigating cardiac and respiratory determinants of heart rate variability in an informationtheoretic framework. Conf Proc IEEE Eng Med Biol Soc. 2014;2014: 6020-6023.

13. Kapidžić A, Platiša MM, Bojić T, Kalauzi A. Nonlinear properties of cardiac rhythm and respiratory signal under paced breathing in young and middle-aged healthy subjects. Med Eng Phys. 2014;36(12): $1577-1584$.

14. Mizuno T, Takahashi T, Cho RY, et al. Assessment of EEG dynamical complexity in Alzheimer's disease using multiscale entropy. Clin Neurophysiol. 2010;121(9):1438-1446.

15. Costa J, González HA, Valldeoriola F, Gaig C, Tolosa E, Valls-Solé J. Nonlinear dynamic analysis of oscillatory repetitive movements in Parkinson's disease and essential tremor. Mov Disord. 2010;25(15):2577-2586.

16. Chen Y, Pham TD. Sample entropy and regularity dimension in complexity analysis of cortical surface structure in early Alzheimer's disease and aging. J Neurosci Methods. 2013;215(2):210-217.

17. Pincus SM, Gladstone IM, Ehrenkranz RA. A regularity statistic for medical data analysis. J Clin Monit. 1991;7(4):335-345.

18. Pincus SM. Approximate entropy as a measure of system complexity. Proc Natl Acad Sci U S A. 1991;88(6):2297-2301. 
19. Richman JS, Moorman JR. Physiological time-series analysis usingapproximate entropy and sample entropy. Am J Physiol Heart Circ Physiol. 2000;278(6):H2039-H2049.

20. Ahmed MU, Mandic DP. Multivariate multiscale entropy: a tool for complexity analysis of multichannel data. Phys Rev E Stat Nonlin Soft Matter Phys. 2011;84(6 Pt 1):061918.

21. Ahmed MU, Li L, Cao J, Mandic DP. Multivariate multiscale entropy for brain consciousness analysis. Conf Proc IEEE Eng Med Biol Soc. 2011;2011:810-813.

22. Bullmore E, Sporns O. Complex brain networks: graph theoretical analysis of structural and functional systems. Nat Rev Neurosci. 2009;10(3): 186-198.

23. Malliani A, Pagani M, Lombardi F, Cerutti S. Cardiovascular neural regulation explored in the frequency domain. Circulation. 1991;84(2): 482-492.

24. Pagani M, Lombardi F, Guzzetti S, et al. Power spectral analysis of heart rate and arterial pressure variabilities as a marker of sympatho-vagal interaction in man and conscious dog. Circ Res. 1986;59(2):178-193.

25. Malliani A, Montano N. Heart rate variability as a clinical tool. Ital Heart J. 2002;3(8):439-445.

26. Bootsma M, Swenne CA, Janssen MJA, Cats VM, Schalij MJ. Heart rate variability and sympathovagal balance: pharmacological validation. Neth Heart J. 2003;11(6):250-259.

27. Li X, Yu S, Chen H, Lu C, Zhang K, Li F. Cardiovascular autonomic function analysis using approximate entropy from 24-h heart rate variability and its frequency components in patients with type 2 diabetes. J Diabetes Investig. 2015;6(2):227-235.

28. Sungnoon R, Muengtaweepongsa S, Kitipawong P, Suwanprasert K, Ngarmukos T. Increased sample entropy in atrial fibrillation relates to cardiac autonomic dysfunction determined by heart rate variability: a preliminary study. In: Biomedical Engineering International Conference (BMEiCON), Ubon Ratchathani; IEEE; 2012;1-4

29. Turianikova Z, Javorka K, Baumert M, Calkovska A, Javorka M. The effect of orthostatic stress on multiscale entropy of heart rate and blood pressure. Physiol Meas. 2011;32(9):1425-1437.

30. Zhang D, She J, Yang J, Yu M. Linear and nonlinear dynamics of heart rate variability in the process of exposure to $3600 \mathrm{~m}$ in $10 \mathrm{~min}$. Australas Phys Eng Sci Med. 2015;38(2):263-270.

31. Cornforth DJ, Tarvainen MP, Jelinek HF. Using Renyi entropy to detect early cardiac autonomic neuropathy. Conf Proc IEEE Eng Med Biol Soc. 2013;2013:5562-5565.

32. Cysarz D, Linhard M, Edelhäuser F, et al. Symbolic patterns of heart rate dynamics reflect cardiac autonomic changes during childhood and adolescence. Auton Neurosci. 2013;178(1-2):37-43.

33. Tang S-C, Jen H-I, Lin Y-H, et al. Complexity of heart rate variability predicts outcome in intensive care unit admitted patients with acute stroke. J Neurol Neurosurg Psychiatry. 2015;86(1):95-100.

34. Chen $\mathrm{CH}$, Huang PW, Tang SC, et al. Complexity of heart rate variability can predict stroke-in-evolution in acute ischemic stroke patients. Sci Rep. 2015;5:17552.

35. Tobaldini E, Nobili L, Strada S, Casali KR, Braghiroli A, Montano N. Heart rate variability in normal and pathological sleep. Front Physiol. 2013;4:294

36. Somers VK, Dyken ME, Mark AL, Abboud FM. Sympathetic-nerve activity during sleep in normal subjects. $N$ Engl J Med. 1993;328(5): 303-307.

37. Vigo DE, Dominguez J, Guinjoan SM, et al. Nonlinear analysis of heart rate variability within independent frequency components during the sleep-wake cycle. Auton Neurosci. 2010;154(1-2):84-88.

38. Virtanen I, Ekholm E, Polo-Kantola P, Huikuri H. Sleep stage dependent patterns of nonlinear heart rate dynamics in postmenopausal women. Auton Neurosci. 2007;134(1-2):74-80.

39. Viola AU, Tobaldini E, Chellappa SL, Casali KR, Porta A, Montano N. Short-term complexity of cardiac autonomic control during sleep: REM as a potential risk factor for cardiovascular system in aging. PLoS One. 2011;6(4):e19002.
40. Yang AC, Tsai S-J, Yang C-H, Kuo C-H, Chen T-J, Hong C-J. Reduced physiologic complexity is associated with poor sleep in patients with major depression and primary insomnia. JAffect Disord. 2011;131(1-3):179-185.

41. Cabiddu R, Trimer R, Borghi-Silva A, et al. Are complexity metrics reliable in assessing HRV control in obese patients during sleep? PLoS One. 2015;10(4):e0124458.

42. Aktaruzzaman M, Migliorini M, Tenhunen M, Himanen SL, Bianchi AM, Sassi R. The addition of entropy-based regularity parameters improves sleep stage classification based on heart rate variability. Med Biol Eng Comput. 2015;53(5):415-425.

43. Burioka N, Miyata M, Cornélissen G, et al. Approximate entropy in the electroencephalogram during wake and sleep. Clin EEG Neurosci. 2005;36(1):21-24.

44. Li H, Peng C, Ye D. A study of sleep staging based on a sample entropy analysis of electroencephalogram. Biomed Mater Eng. 2015;26(suppl 1):S1149-S1156

45. Thul A, Lechinger J, Donis J, et al. EEG entropy measures indicate decrease of cortical information processing in disorders of consciousness. Clin Neurophysiol. 2016;127(2):1419-1427.

46. Lee GM, Fattinger S, Mouthon AL, Noirhomme Q, Huber R. Electroencephalogram approximate entropy influenced by both age and sleep. Front Neuroinform. 2013;7:33.

47. Chung C-C, Kang J-H, Yuan R-Y, et al. Multiscale entropy analysis of electroencephalography during sleep in patients with Parkinson disease. Clin EEG Neurosci. 2013;44(3):221-226.

48. Abásolo D, Hornero R, Espino P, Poza J, Sánchez CI, de la Rosa R. Analysis of regularity in the EEG background activity of Alzheimer's disease patients with approximate entropy. Clin Neurophysiol. 2005;116(8):1826-1834.

49. Abásolo D, Hornero R, Espino P, Escudero J, Gómez C. Electroencephalogram background activity characterization with approximate entropy and auto mutual information in Alzheimer's disease patients. Conf Proc IEEE Eng Med Biol Soc. 2007;2007:6192-6195.

50. Abásolo D, Hornero R, Espino P, Alvarez D, Poza J. Entropy analysis of the EEG background activity in Alzheimer's disease patients. Physiol Meas. 2006;27(3):241-253.

51. Jeong J. EEG dynamics in patients with Alzheimer's disease. Clin Neurophysiol. 2004;115(7):1490-1505.

52. Stam CJ. Nonlinear dynamical analysis of EEG and MEG: review of an emerging field. Clin Neurophysiol. 2005;116(10):2266-2301.

53. Escudero J, Abásolo D, Hornero R, Espino P, López M. Analysis of electroencephalograms in Alzheimer's disease patients with multiscale entropy. Physiol Meas. 2006;27(11):1091-1106.

54. Park J-H, Kim S, Kim C-H, Cichocki A, Kim K. Multiscale entropy analysis of EEG from patients under different pathological conditions. Fractals. 2007;15(4):399-404.

55. Yang AC, Wang S-J, Lai K-L, et al. Cognitive and neuropsychiatric correlates of EEG dynamic complexity in patients with Alzheimer's disease. Prog Neuropsychopharmacol Biol Psychiatry. 2013;47:52-61.

56. Delbeuck X, Van der Linden M, Collette F. Alzheimer's disease as a disconnection syndrome? Neuropsychol Rev. 2003;13(2):79-92.

57. Toussaint P-J, Maiz S, Coynel D, et al. Characteristics of the default mode functional connectivity in normal ageing and Alzheimer's disease using resting state fMRI with a combined approach of entropybased and graph theoretical measurements. Neuroimage. 2014;101: 778-786.

58. Fabris C, Sparacino G, Sejling A-S, et al. Hypoglycemia-related electroencephalogram changes assessed by multiscale entropy. Diabetes Technol Ther. 2014;16(10):688-694.

59. Giannakakis G, Sakkalis V, Pediaditis M, Farmaki C, Vorgia P, Tsiknakis M. An approach to absence epileptic seizures detection using approximate entropy. Conf Proc IEEE Eng Med Biol Soc. 2013;2013: 413-416.

60. Taghavi M, Boostani R, Sabeti M, Taghavi SMA. Usefulness of approximate entropy in the diagnosis of schizophrenia. Iran J Psychiatry Behav Sci. 2011;5(2):62-70. 
61. Bachiller A, Lubeiro A, Díez A, et al. Decreased entropy modulation of EEG response to novelty and relevance in schizophrenia during a P300 task. Eur Arch Psychiatry Clin Neurosci. 2015;265(6):525-535.

62. Kreuzer M, Kochs EF, Schneider G, Jordan D. Non-stationarity of EEG during wakefulness and anaesthesia: advantages of EEG permutation entropy monitoring. J Clin Monit Comput. 2014;28(6):573-580.

63. Liang Z, Wang Y, Sun X, et al. EEG entropy measures in anesthesia. Front Comput Neurosci. 2015;9:16.

64. Olanow CW, Tatton WG. Etiology and pathogenesis of Parkinson's disease. Annu Rev Neurosci. 1999;22:123-144.

65. Darbin O, Adams E, Martino A, Naritoku L, Dees D, Naritoku D. Nonlinear dynamics in parkinsonism. Front Neurol. 2013;4:211.

66. Pelykh O, Klein A-M, Bötzel K, Kosutzka Z, Ilmberger J. Dynamics of postural control in Parkinson patients with and without symptoms of freezing of gait. Gait Posture. 2015;42(3):246-250.

67. Han C-X, Wang J, Yi G-S, Che Y-Q. Investigation of EEG abnormalities in the early stage of Parkinson's disease. Cogn Neurodyn. 2013;7(4): 351-359.

68. Darbin O, Dees D, Martino A, Adams E, Naritoku D. An entropy-based model for basal ganglia dysfunctions in movement disorders. Biomed Res Int. 2013;2013:742671.

69. Mei Z, Zhao G, Ivanov K, et al. Sample entropy characteristics of movement for four foot types based on plantar centre of pressure during stance phase. Biomed Eng Online. 2013;12:101

70. Jiang BC, Yang W-H, Shieh J-S, Fan JS-Z, Peng C-K. Entropy-based method for COP data analysis. Theor Issues Ergon Sci. 2013;14(3): 227-246.

71. Pansera M, Estrada JJ, Pastor L, Cancela J, Greenlaw R, Arredondo MT. Multi-parametric system for the continuous assessment and monitoring of motor status in Parkinson's disease: an entropy-based gait comparison. Conf Proc IEEE Eng Med Biol Soc. 2009;2009:1242-1245.

72. McGregor SJ, Busa MA, Skufca J, Yaggie JA, Bollt EM. Control entropy identifies differential changes in complexity of walking and running gait patterns with increasing speed in highly trained runners. Chaos. 2009;19(2):026109.
73. Costa M, Priplata AA, Lipsitz LA, et al. Noise and poise: enhancement of postural complexity in the elderly with a stochastic-resonance-based therapy. Europhys Lett. 2007;77:68008.

74. Kang HG, Costa MD, Priplata AA, et al. Frailty and the degradation of complex balance dynamics during a dual-task protocol. J Gerontol A Biol Sci Med Sci. 2009;64(12):1304-1311.

75. Manor B, Costa MD, Hu K, et al. Physiological complexity and system adaptability: evidence from postural control dynamics of older adults. J Appl Physiol (1985). 2010;109(6):1786-1791.

76. Cavanaugh JT, Mercer VS, Stergiou N. Approximate entropy detects the effect of a secondary cognitive task on postural control in healthy young adults: a methodological report. J Neuroeng Rehabil. 2007;4:42.

77. Raja Beharelle A, Kovačević N, McIntosh AR, Levine B. Brain signal variability relates to stability of behavior after recovery from diffuse brain injury. Neuroimage. 2012;60(2):1528-1537.

78. Sokunbi MO, Gradin VB, Waiter GD, et al. Nonlinear complexity analysis of brain FMRI signals in schizophrenia. PLoS One. 2014;9(5):e95146.

79. Yang AC, Huang C-C, Yeh H-L, et al. Complexity of spontaneous BOLD activity in default mode network is correlated with cognitive function in normal male elderly: a multiscale entropy analysis. Neurobiol Aging. 2013;34(2):428-438.

80. Lin P-F, Lo M-T, Tsao J, Chang Y-C, Lin C, Ho Y-L. Correlations between the signal complexity of cerebral and cardiac electrical activity: a multiscale entropy analysis. PLoS One. 2014;9(2):e87798.

81. Chen M-S, Jiang BC. Resistance training exercise program for intervention to enhance gait function in elderly chronically ill patients multivariate multiscale entropy for center of pressure signal analysis. Comput Math Methods Med. 2014;2014:471356.

82. Wayne PM, Gow BJ, Costa MD, et al. Complexity-based measures inform effects of Tai Chi training on standing postural control: crosssectional and randomized trial studies. PLoS One. 2014;9(12):e114731.

83. Zhou D, Zhou J, Chen H, Manor B, Lin J, Zhang J. Effects of transcranial direct current stimulation (tDCS) on multiscale complexity of dual-task postural control in older adults. Exp Brain Res. 2015;233(8):2401-2409.
Neuroscience and Neuroeconomics

\section{Publish your work in this journal}

Neuroscience and Neuroeconomics is an international, peer-reviewed, open access journal focusing on the identification of brain structures and measurement of neural activity related to behavior, behavioral predictions, and decision making in health and disease. The manuscript management system is completely online and includes a very quick and fair peer-review system. Visit http://www.dovepress.com/testimonials. php to read real quotes from published authors. 